\title{
Potential associations between alterations in gut microbiome and obesity- related traits after the bariatric surgery
}

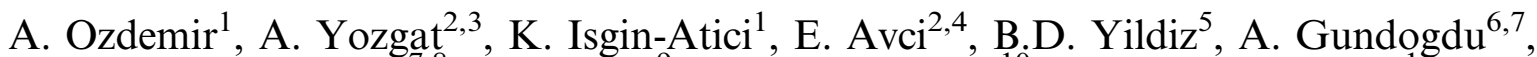 \\ U. Nalbantoglu ${ }^{7,8}$, T. Turhan ${ }^{9}$, F. Dogruman-A1 ${ }^{10}$ and Z. Buyuktuncer ${ }^{1}$ \\ ${ }^{1}$ Department of Nutrition and Dietetics, Faculty of Health Sciences, Hacettepe University, Ankara, Turkey, \\ ${ }^{2}$ Department of Gastroenterology, Ankara Numune Research and Education Hospital, Ankara, Turkey, \\ ${ }^{3}$ Department of Gastroenterology, School of Medicine, Ufuk University, Ankara, Turkey, \\ ${ }^{4}$ Department of Gastroenterology, Medicana Hospital, Konya, Turkey, \\ ${ }^{5}$ Department of General Surgery, Ankara Numune Research and Education Hospital, Ankara, Turkey, \\ ${ }^{6}$ Department of Microbiology and Clinical Microbiology, School of Medicine, Erciyes University, Kayseri, Turkey, \\ ${ }^{7}$ Genome and Stem Cell Center, Erciyes University, Kayseri, Turkey, \\ ${ }^{8}$ Department of Computer Engineering, School of Engineering, Erciyes University, Kayseri, Turkey, \\ ${ }^{9}$ Department of Biochemistry, Ankara Numune Research and Education Hospital, Ankara, Turkey and \\ ${ }^{10}$ Department of Medical Microbiology, School of Medicine, Gazi University, Ankara, Turkey
}

Bariatric surgery can provide weight loss through not only reducing dietary intake but also modifying metabolic control, hungersatiety mechanisms and gut microbiome ${ }^{(1-4)}$. This study investigated the effects of bariatric surgery on dietary intake, gut microbiome and metabolic markers.

The study included 15 morbid obese who had bariatric surgery after baseline interview (Study Group), 8 morbid obese (Control group-1) and 11 non-obese individuals (Control group-2). All participants were followed-up for a 6-month period with face-to-face interviews at baseline (M0), at the end of 3 (M3) and 6 months (M6). Nutritional assessment including dietary intake and anthropometry was done, blood samples were taken for biochemical analysis, and faecal samples were collected for gut microbiome analysis at M0, M3 and M6. Microbiota analysis was performed using QIIME pipeline. Kruskal-Wallis, Wilcoxon and Spearman's rank correlation tests were performed with SPSS 23.0.

Participants in the study group lost about $33 \mathrm{~kg}$ of body weight in 6 months, in addition to declines in other anthropometric measurements $(p<0.05$, for each). Dietary energy and nutrient intakes decreased immediately after surgery. Serum glucose, total cholesterol, LDL-cholesterol and hs-CRP levels decreased by surgery $(\mathrm{p}<0.05$, for each). No changes in anthropometrical measurements, dietary intake or biochemical parameters were observed in any of control groups during follow-up period ( $\mathrm{p}>0.05$, for each). Morbidly obese participants had higher levels of Firmicutes and lower levels of Bacteroidetes than non-obese subjects at the baseline. By the surgery, the abundances of phylum Bacteroidetes increased from $27.9 \%$ to $46.0 \%(\mathrm{p}=0.02)$ and phylum Firmicutes decreased from $50.3 \%$ to $41.7 \%(p>0.05)$, leading a significant decrease in Firmicutes/Bacteroidetes ratio ( $p=0.01)$ from M0 to M6. At genus level, abundance of Lactobacillus and Bifidobacterium decreased while Akkermansia increased at M6 in study group ( $p<0.01$, for each). In terms of diversity, morbidly obese participants had a distinct profile according to B-diversity indices at M0. However, it changed towards the profile of non-obese participants at M3, and became similar with them at M6 ( $p>0.05$ ) in study group. Besides, $\alpha$-diversity indices were lower in morbidly obese subjects at M0, but became similar to levels in non-obese controls at M6. No correlation between the alterations in biochemical parameters and relative abundances of Bacteroidetes and Firmicutes phyla was observed, however, decline in serum total and LDL cholesterol levels were negatively correlated with decrease in the relative abundance of Proteobacteria $(r=-0.718, p=0.013)$. Furthermore, decrease in the abundance of Firmicutes positively correlated with the changes in both total fat and monounsaturated fatty acid intakes $(r=0.575, \mathrm{p}=0.025)$.

This study confirmed that bariatric surgery has substantial impacts on gut microbiome profile and diversity, as well as biochemical parameters, which were associated with the alterations in dietary intake patterns.

\section{Acknowledgements}

This study was funded by Hacettepe University Scientific Research Projects Coordination Unit.

\section{References}

1. Wing RR \& Phelan S (2005) Am J Clin Nutr 82(1), 222-225

2. Buchwald H, Avidor Y, Braunwald E et al. (2004) JAMA 292(14), 1724-1737.

3. Sinclair P, Brennan DJ \& le Roux CW (2018) Nat Rev Gastroenterol Hepato 15(10), 606-624.

4. Knop FK \& Taylor R (2013) Diabetes Care 36(2), 287-291. 